Results Among 2678 recreational and daily living boating victims during 1991-2010, included were infants less than 1 year old 4 , toddlers 1-4 years old 18, 5-14 year olds 57 and youth 15-19 years old 219. By sex, all 4 infants were male, 13 toddlers male and 5 female, 40 of 5-14s male and 17 female, and 198 youth male and 21 female. $20 \%$ of $0-14$-year-old victims were properly wearing a PFD, compared with $11 \%$ for youth 15-19 years. For $35 \%$ of child boaters, there was reportedly no PFD present, representing a violation of current legislation, and for another $27 \%$ it was unknown whether a PFD was present. Hence as many as $62 \%$ were in violation. For indigenous children, in 63\% no PFD was present, and for $17 \%$ unknown, i.e., as high as 80 s in violation, compared with other ethnicities where $21 \%$ were not present and 26\% unknown. Concerning youth, for 40\% no PFD was present, and for $28 \%$ unknown, hence as high as $68 \%$ in violation. Among indigenous youth, 58\% had no PFD present and $36 \%$ unknown, i.e., as high as $92 \%$ in violation. For other ethnicities, 41\% had no PFD present and 26\% unknown. 33\% of 014-year-old non-indigenous victims were properly wearing a PFD, and $13 \%$ of $15-19$-year-olds. Not a single aboriginal victim 0-19 years was properly wearing a PFD during the 20 year period, compared with $18 \%$ for other ethnicities. $25 \%$ of victims 0-14 years were alone or with minors only. For the remaining $75 \%$, at least one adult was present. For 15-19-year-olds, 20\% were alone or with minors only, $65 \%$ with at least one adult and $13 \%$ with others. Of the 17 victims $0-19$ years alone at the time of the incident, only one was wearing a PFD. For 0-14 year olds with an adult, 25\% were properly wearing a PFD, and for 15-19 year olds, $13 \%$.

Conclusions Wearing of a flotation device was low among all child and youth immersion victims. Non-wearing was markedly lower among indigenous victims. Special effort is needed to address non-wearing and lack of compliance with regulations among indigenous peoples of Canada.

\section{THE TOTAL SERVICE PLAN: CASE STUDIES HIGHLIGHT HOW EVIDENCE IS USED TO SHAPE POLICY AND INFORM INTERVENTION STRATEGIES}

Barbara Brighton. Surf Life Saving Australia

\subsection{6/injuryprev-2016-042156.268}

Background In an effort to reduce drowning deaths Surf Life Saving Australia (SLSA) has created the Total Service Plan to develop the national coastal safety strategy. A central tenet of the plan is that SLSA is a knowledge and research hub with a focus on nationally significant issues and programs.

Key items included in and driven by the plan are:

- Research and data

- National Safety Agenda

- Operations, including distribution of resources and services

- Public education

The Total Service Plan is created using an iterative process of data analysis and review to identify coastal safety issues of national importance. It follows the public health model and is aligned with international risk management principles.

Methods At the core of the plan is the data, including existing material such as population and drowning data, rescue statistics and operational data, as well coastal risk assessments. SLSA also uses new data, for example, a recent National Coastal Safety Survey, which explored attitudes and behaviours of the Australian public regarding the coast and safety.

In collaboration with stakeholders, the Coastal Safety team analyses this information to identify and prioritise national safety issues and priorities as well as drowning blackspots. The issues and blackspots identified through this process form SLSA's National Safety Agenda.

Monitoring and evaluation is built into the Total Service Plan. Each component is reviewed, evaluated, revised and updated as new evidence and data become available. Every program or project is regularly assessed and improved upon or discontinued as the case may be.

Results The National Safety Agenda influences and prioritises lifesaving operations, including services and equipment allocation, such as introducing lifesaving patrols during the wet season in Darwin, NT, to reduce drowning deaths and decrease the incidents of box jellyfish stings and crocodile attacks.

It also drives public education including evidence-based mitigation strategies, communications campaigns and pilot projects. For example, funding a community education project in Wanneroo, WA, to build residents' knowledge and awareness of specific water safety hazards to help increase their resilience to coastal hazards and ultimately reduce drowning deaths.

Conclusions This presentation will investigate case studies from the Total Service Plan to show how SLSA uses evidence to ensure lifesaving services and assets are located in areas of need and appropriate public education programs and mitigation strategies are in place to address coastal safety issues and known drowning blackspots.

\section{EVOLUTION OF THE CHILD DROWNING PREVENTION PROGRAMME IN THAILAND}

Suchada Gerdmongkolgan, Som Ekchaloemkiet. Bureau of Non-Communicable Diseases, Department of Disease Control, Ministry of Public Health, Thailand

\subsection{6/injuryprev-2016-042156.269}

Background Drowning is the number one cause of death among children under 15 years of age in Thailand, taking into account all deaths due to infectious and non-infectious diseases. Child drowning death rate (per 100,000 child population) ranged from 6.8 to 11.5 between 2004 and 2014. The Child Drowning Prevention Programme in Thailand has been implemented since late 2006 with the goal of reducing the child drowning death rate to 5.0 by 2018 . The Objective of this study is to develop the standards for the operations of the Child Drowning Prevention Programme in Thailand.

Methods The operations of the Child Drowning Prevention Programme in Thailand during the period 2006-2014 were reviewed and then a gap analysis was conducted for use as a guide for developing the standards, or bridging the gap, for the operations of the programme.

Results After implementing several of such measures, the child drowning death rate declined constantly from 11.5 in 2005 (the first year of programme implementation) to 6.8 in 2014. But gaps were noted at the local or community level, such as the lack of continuity, encouragement and operations of in all aspects of 
the programme. Thus, to bridge the gaps, in 2015 the Ministry of Public Health adopts the "Merit Maker for Child Drowning Prevention" measure, aiming to identify the networks working on drowning prevention that covers at least six key measures, emphasising community-based multi-disciplinary approach, resource sharing, participation of local agencies, and rewarding Merit Makers whose contributions are in accordance with the established criteria.

Conclusion Since the launch of the programme on child drowning prevention, policies and measures have been implemented, resulting in a constant reduction in child drowning fatalities. In such efforts, the Ministry of Public Health has also revised several measures so that they are more effective in the country in achieving the child drowning reduction goal.

\section{SURVIVAL SWIMMING - EFFECTIVENESS OF SWIMSAFE IN PREVENTING DROWNING IN MID AND LATE CHILDHOOD}

${ }^{1,2}$ Abu Talab, ${ }^{1,2}$ Aminur Rahman, 1,2 Fazlur Rahman, ${ }^{1,2}$ Jahangir Hossain, ${ }^{3}$ Justin Scarr, ${ }^{4}$ Michael Linnan. ${ }^{1}$ International Drowning Research Centre - Bangladesh (IDRCB); ${ }^{2}$ Centre for Injury Prevention and Research, Bangladesh (CIPRB); ${ }^{3}$ Royal Life Saving Society Australia; ${ }^{4}$ the Alliance for Safe Children (TA SC)

\subsection{6/injuryprev-2016-042156.270}

Introduction Conclusive evidence of the protective effect of swimming has been lacking in high income countries. Nested case control studies in surveys done across Asia have shown significant associations between swimming ability and protection against drowning in children four years and older. In order to confirm that the relationship between swimming ability and protection from drowning was a causal relationship, a four year cohort study of a structured survival swimming program known as SwimSafe was undertaken in Bangladesh.

Methods A cohort study was conducted to compare the protection from drowning conferred by participation in the SwimSafe survival swimming. The survival swimming cohort was made up of children primarily aged 4-12 years old who were graduates of the SwimSafe program in rural Bangladesh. The non-survival swimming cohort was made up of age- and sex-matched children from neighbouring villages who were not participants in the SwimSafe program. Death from drowning was compared in both groups using survival analysis techniques.

Results A total of 81,659 children had been taught survival swimming in the study area who had been followed through September 30, 2010 and whose survival status was known. A cohort of 140,479 non-SwimSafe participants had been followed in the same system and whose survival status was known. Children from this non-SwimSafe cohort were matched on age and sex with SwimSafe graduates and a total of 66,066 children from each cohort were fully matched. The matching cohorts were subjected to Kaplan Meier and Cox Proportional Hazard survival analyses with fatal drowning as the primary outcome. These showed a substantial and statistically significant reduction in relative risk of drowning in SwimSafe children as compared to the matched non-SwimSafe children.

Conclusions Survival swimming, as taught in the SwimSafe survival swimming program with its highly structured and skills based methodology confers protection from drowning in children who graduate from the SwimSafe program.

\section{UNINTENTIONAL DROWNING AND PSYCHOACTIVE SUBSTANCES}

${ }^{1}$ Tuulia Pajunen, ${ }^{1}$ Philippe Lunetta, ${ }^{2}$ Gordon Smith, ${ }^{3}$ Erkki Vuori, ${ }^{4}$ Pirjo Lillsunde, ${ }^{5}$ Frank F Vincenzi. ' University of Turku, Finland; ${ }^{2}$ University of Maryland, USA; ${ }^{3}$ University of Helsinki, Finland; ${ }^{4}$ National Institute for Health and Welfare, Finland; ${ }^{5}$ University of Washington, USA

\subsection{6/injuryprev-2016-042156.271}

Background Alcohol is a well-known risk factor in unintentional drownings. While psychotropic drugs may also cause psychomotor impairment and affect cognition, no detailed studies have focused on their association with drowning. Finland provides extensive post-mortem toxicological data for studies on drowning due to its high medico-legal autopsy rates.

Methods Drowning cases, 2000 through 2009, for which postmortem toxicological analysis was performed, were selected from the database of Toxicological laboratory, Department of Forensic Medicine, University of Helsinki, using the ICD-10 nature of injury code T75.1. The data were narrowed down to unintentional drowning, using the ICD-10 external injury codes V90, V92 and W65-74. For each drowning case, the blood alcohol concentration (BAC) and the concentrations of other drugs were recorded. The contribution of psychotropic drugs to drowning was evaluated based on their blood concentration by mean of a 6-grade scale.

Results Among victims $\geq 15$ years old, there were 1697 unintentional drownings. $303(17.8 \%)$ were boating-related and 1394 $(82.2 \%)$ non-boating-related. $65.0 \%$ of boating-related and $61.8 \%$ of non-boating-related victims were alcohol-positive $(=\mathrm{BAC} \geq 0.5 \%$ ) $)$. The male to female ratio in alcohol-positive drownings was 7.3. At least one psychotropic drug was found in $453(26.7 \%)$ drowning cases, with some victims having up to 7 different drugs. Overall 70 different psychotropic drugs were detected. 134 (7.9\%) cases were both alcohol-negative and psychotropic drug -positive, of them 59 (3.5\%) were graded 4 to 6 , indicating possible to very probable contribution to drowning. Conclusions Psychotropic drugs alone or in association with alcohol may be an overlooked risk factor to drowning due to their effects on psychomotor function and cognition. Future studies should also address other mechanisms, for instance druginduced long QT-syndrome, by which drugs may contribute to drowning.

\section{EVALUATION OF MEASURES FOR THE MANAGEMENT OF HIGH-RISK WATER SOURCES IN COMMUNITIES FOR CHILD DROWNING PREVENTION IN THAILAND}

Suchada Gerdmongkolgan, Som Ekchaloemkiet. Department of Diseases Control, Ministry of Public Health, Thailand

\subsection{6/injuryprev-2016-042156.272}

Background In Thailand, drowning is the number one cause of death among children under 15 years of age, whose drowning death rate ranged from 7.6 to 11.5 per 100,000 child population between 2004 and 2013 .

Objective To evaluate the measures for the management of highrisk water sources in communities by installing warning signboards and easily available water safety devices for child drowning prevention.

Methods This evaluative research was conducted in two types of communities: one with the installation of warning signboards on water safety and advice for assisting drowning victims and 